\title{
PARTITIONING OF CERRENA UNICOLOR LACCASE ACTIVITY IN AN AQUEOUS TWO-PHASE SYSTEM
}

\author{
Michał Blatkiewicz" ${ }^{* 1}$ Axel Prinz ${ }^{2}$, Andrzej Górak ${ }^{1,2}$, Stanisław Ledakowicz ${ }^{1}$ \\ ${ }^{1}$ Lodz University of Technology, Faculty of Process and Environmental Engineering, ul. \\ Wólczańska 213, 90-942, Łódź, Poland \\ ${ }^{2}$ Dortmund University of Technology, The Department of Biochemical and Chemical Engineering, \\ Emil-Figge-Straße 70, D-44227 Dortmund, Germany
}

\begin{abstract}
Culture supernatant containing laccase produced by Cerrena unicolor strain was used to examine laccase partitioning between phases in an aqueous two-phase system. The investigated system consisted of polyethylene glycol 3000 and sodium phosphate buffer adjusted to $\mathrm{pH}=7$. Influence of several parameters on partitioning was measured, including phase forming components' concentrations, tie line lengths, phase volume ratio, supernatant dilution, process temperature and halogen salt supplementation. Partitioning coefficients up to 78 in the bottom phase were achieved with yields of over $90 \%$. Tie line length and phase volume ratio had significant effect on enzyme partitioning.
\end{abstract}

Keywords: ATPS, laccase, downstream processing

\section{INTRODUCTION}

Laccases (EC 1.10.3.2, p-diphenolic oxidases) are enzymes which belong to the oxidoreductase group. Their structure molecules contain multiple copper atoms. They are produced mostly by fungi and higher plants (Harvey et al., 1999), but there have been reports of laccase-like enzymes found in bacteria and insects (Claus and Filip, 1997; Diamantidis et al., 2001). Laccases were first discovered by Yoshida (Yoshida, 1883), and are mostly known for their ability to decompose lignin chains. However, the spectrum of chemical compounds reduced by the enzyme is very broad, including substituted phenols, aromatic amines and thiols (Xu, 1996).

Cerrena unicolor is a white rot Basidiomycete (Enebak and Blanchette, 1989). It produces relatively high amount of laccase, without the need of additional inductors, unlike other fungal strains (Rogalski et al., 1999), which makes it an attractive species for laboratory and industrial production of the enzyme.

Downstream processing of biologically obtained macromolecules remains the bottleneck of the majority of processes applying biotechnology. According to Kula et al. (1982), about $60-80 \%$ of all expenses in biotechnological processes are spent on downstream processing. Various conventional methods, such as micro- and ultrafiltration (Bryjak and Rekuć, 2009), chromatography (Yang et al., 2013), or precipitation (Rajeeva and Lele, 2010), have been employed for downstream processing of laccases. Unfortunately, those methods are highly inefficient in terms of yields and energy consumption. One of the most promising methods for laccase purification is aqueous two-phase 
extraction (ATPE), which allows enzyme concentration up to several hundredfold with good selectivity and recoveries up to 95\% (Johansson and Reczey, 1998).

One of the main problems in biochemical industry is the fact that many biochemical products as proteins are prone to denaturation when exposed to most organic solvents. One of the methods to overcome this problem is using aqueous two-phase systems (ATPS) for protein purification, because of their ability to extract macromolecules without deactivating them, the ease of scale-up and usually high activity yields and partitioning coefficients (Diamond and Hsu, 1989; Silvério et al., 2012). Aqueous two-phase systems consist of two non-miscible aqueous phases, which are formed when two mutually immiscible polymers, or a polymer with a salt, an alcohol and a salt, an ionic liquid and a salt, or two surfactants solutions are introduced into the system (Salabat et al., 2010). Their water content ranges between 70\% and 90\% (Ratanapongleka and Phetsom, 2011). A schematic phase diagram is shown in Fig. 1.

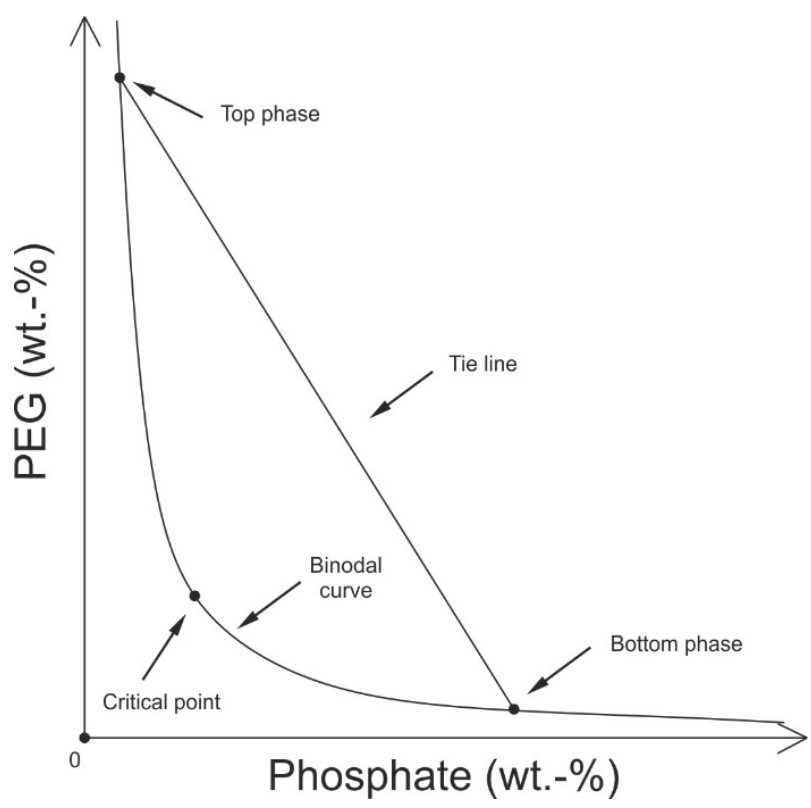

Fig. 1. An aqueous two-phase system phase diagram

Such a method of protein extraction is a very complex phenomenon, and it is difficult to model, due to various factors that influence the partitioning of the solute, such as its hydrophobicity, molecular size, electrochemical properties, molecular conformation, biospecificity, and also the properties of the ATPS, such as the phase-forming components, $\mathrm{pH}$, temperature and ionic strength (Azevedo et al., 2009), although models of specific phenomena, such as phase forming (Reschke et al., 2014), or partitioning of specific molecules (Pazuki et al., 2009) have been created. Therefore, each proteinATPS combination can be treated as unique and requiring its own experimental data. The aim of this paper is to determine the influence of various process parameters on Cerrena unicolor laccase activity in PEG-phosphate aqueous two-phase systems.

\section{MATERIALS AND METHODS}

\subsection{Materials}

The Cerrena unicolor fungus was first grown on agar plates. After 7 days of growth, the culture was moved into 200ml flasks, containing Lindeberg-Holm growth medium (Lindeberg and Holm, 1952). The liquid fermentation was conducted for 10 days in a shaker at $110 \mathrm{rpm}$ and the temperature of 
$26{ }^{\circ} \mathrm{C}$. Then, the supernatant was filtrated and frozen in $50 \mathrm{ml}$ falcon tubes at $-20^{\circ} \mathrm{C}$. The defrosted supernatant showed an initial activity of about $1500 \mathrm{U} / 1$ in relation to ABTS at $\mathrm{pH}=5.7$ in $37^{\circ} \mathrm{C}$.

For bioproduct recovery, PEG/phosphate systems are recommended (Benavides and Rito-Palomares, 2008; Mayolo-Deloisa et al., 2009). Polyethylene glycol with the molecular weight of $3000 \mathrm{~g} / \mathrm{mol} \mathrm{Ph}$. Eur. Grade (PEG3000) was purchased from Merck (cat. \#34042000). Di-potassium hydrogen phosphate trihydrate $\left(\mathrm{K}_{2} \mathrm{HPO}_{4} \cdot 3 \mathrm{H}_{2} \mathrm{O}\right)$ was purchased from AppliChem (cat. A4001) with the purity of 99\%. Sodium dihydrogen phosphate dehydrate $\left(\mathrm{NaH}_{2} \mathrm{PO}_{4} \cdot 2 \mathrm{H}_{2} \mathrm{O}\right) \mathrm{Ph}$. Eur. Grade $(99.8 \%)$ was purchased from VWR Prolabo (cat. \#28014.360). Sodium chloride $(\mathrm{NaCl})$ was purchased from Sigma Aldrich (cat. \#A4001) with the purity of 99.5\%. 2,2'azino-bis-(3-ethylobenzothiazoline-6-sulphonic acid) (ABTS) with the purity of 98\% was purchased from Sigma-Aldrich (cat. \#A1888).

The water for all dilutions and experiments was purified using the Milli-Q Synthesis A10 system (Millipore, conductivity $\kappa \leq 0.06 \mu \mathrm{S} / \mathrm{cm}$ ).

For all the experiments, stock solutions of 50 wt.- $\%$ of PEG3000 and 29 wt.- $\%$ of phosphate anion $\left(\mathrm{PO}_{4}{ }^{3-}\right)$ were prepared. The $\mathrm{pH}$ of the phosphate solution was equal to 7 .

\subsection{Activity measurements}

The activity of every sample was measured using the ABTS assay analogous to that introduced by Majcherczyk et al. (Majcherczyk et al., 2998). It was determined by the oxidation rate of ABTS molecules over time. According to Luterek et al. (1998) the highest activity of the enzyme is achieved at the $\mathrm{pH}$ between 5.3 and 5.8. Therefore, $\mathrm{pH}=5.7$ sodium acetic acid buffer was prepared. Due to high activity of the enzyme, each sample was diluted in water, with the dilution factor $D=20$. All the measurements were performed in triplicates using a Multiskan FC well plate reader from Thermo Scientific. For each measurement $V_{s}=50 \mu \mathrm{l}$ of the sample was used, and its $\mathrm{pH}$ adjusted to 5.7 by adding $150 \mu \mathrm{l}$ of the buffer. Then, $50 \mu 1$ of $5 \mathrm{mM}$ ABTS solution was added. Therefore, the volume of every measured sample was equal to $V_{t}=250 \mu$, and its height in the cavity equal to $d=6.3 \mathrm{~mm}$. The measurements of absorbance change $\Delta E$ were performed in $37{ }^{\circ} \mathrm{C}$ at $420 \mathrm{~nm}$ wavelength over 3 minutes. The extinction coefficient for ABTS at $420 \mathrm{~nm}$ wavelength is equal to $\varepsilon=0.04321$ $1 /(\mathrm{mmol} \mathrm{cm})$. The activity was defined as the amount of the enzyme catalysing one $\mu \mathrm{mol}$ of ABTS per minute. To calculate it, the following equation was used:

$$
\operatorname{act}\left[\frac{U}{l}\right]=\frac{\Delta E \cdot D \cdot V_{t}}{\Delta t \cdot V_{s} \cdot d \cdot \varepsilon}
$$

For every sample the assay was performed in triplicates, and standard deviations between results were calculated. The standard deviations, as well as the errors of the weighing scale and the burette were used for Gaussian error propagation.

\subsection{Experimental setup}

ATPE experiments were conducted in the same vessels described by Prinz et al. (2012). The flasks consisted of extraction vessels combined with burettes of approximately $20 \mathrm{ml}$ volume.

Mixture components, in the form of stock solutions and culture supernatant, were transferred into the vessels with the use of automatic pipettes, and the sample was complemented with millipore water to reach the mass of $15 \mathrm{~g}$ per sample. Then the flasks were closed and sealed with parafilm. The mixing was carried out in a tempered water tank equipped with a magnetic stirrer at $25{ }^{\circ} \mathrm{C}$. The samples were stirred for an hour at 300rpm to achieve phase equilibrium. 
After the mixing, the stirrer was turned off and the mixtures were left in the same position for the initial phase settling. After an hour, the flasks were carefully turned upside-down to transfer the phases to the burette part. In such position, the vessels were stored in the same tank for approximately twenty hours in order to separate the phases: the salt-rich bottom phase (BP) and the polymer-rich top phase (TP). Such long time was needed because of low density differences and low surface tensions between phases (Kim and Rha, 2000).

Finally, the flasks were removed from the tank, and the phases were separated into different vessels and weighed. To prevent further, temperature-induced separation of the samples, they were additionally diluted with water before activity measurements.

\section{RESULTS AND DISCUSSION}

The basic parameter to characterise the extraction was the partitioning coefficient $K$. In this case it defined the ratio of activity partitioning between the phases. Since Cerrena unicolor laccase was observed to partition mostly to the hydrophilic phosphate bottom phase, it was defined as activity in the bottom phase divided by the activity of the top phase (2).

$$
K_{a c t}=\frac{a c t_{B P}}{a c t_{T P}}
$$

Another basic parameter was the volume phase ratio, defined as quotient between bottom phase and top phase volumes (3).

$$
\phi=\frac{V_{B P}}{V_{T P}}
$$

An important parameter to describe mixing points of an extraction was the system's tie line length (TLL) (4). It depended on the amount of phase forming components in the mixture. Concentrations of phosphate and PEG in the respective phases were determined with the use of a mathematical model (Prinz et al., 2014), the accuracy of which was tested with ion chromatography experiments.

$$
T L L=\sqrt{\left(x_{P E G / T P}-x_{P E G / B P}\right)^{2}+\left(x_{P h o s / T P}-x_{P h o s / B P}\right)^{2}}
$$

To measure the enzyme activity recovery in each phase, the recovery factor $Y$ (5) was introduced. It determined the activity in a given phase in relation to the initial activity of the enzyme in the culture supernatant.

$$
Y_{i}=\frac{V_{i} \cdot a c t_{i}}{V_{i n i} \cdot a c t_{i n i}} \cdot 100 \%
$$

In this case, the index $i$ referred to the top phase or bottom phase. Although this parameter was expressed in percent, the values in one system did not necessarily add up to $100 \%$, because phase forming components can have significant activating or deactivating influence on the enzyme.

\subsection{Phase forming components}

Before starting the extraction experiments, the influence of phase forming components on the enzyme's stability had to be investigated. The culture supernatant of Cerrena unicolor laccase was therefore mixed with different concentrations of PEG3000 and a phosphate buffer. Each sample was kept in room temperature for a specified time of twenty-four hours. The activity measurements were performed after one hour, three hours, and twenty-four hours after the mixtures had been created. 


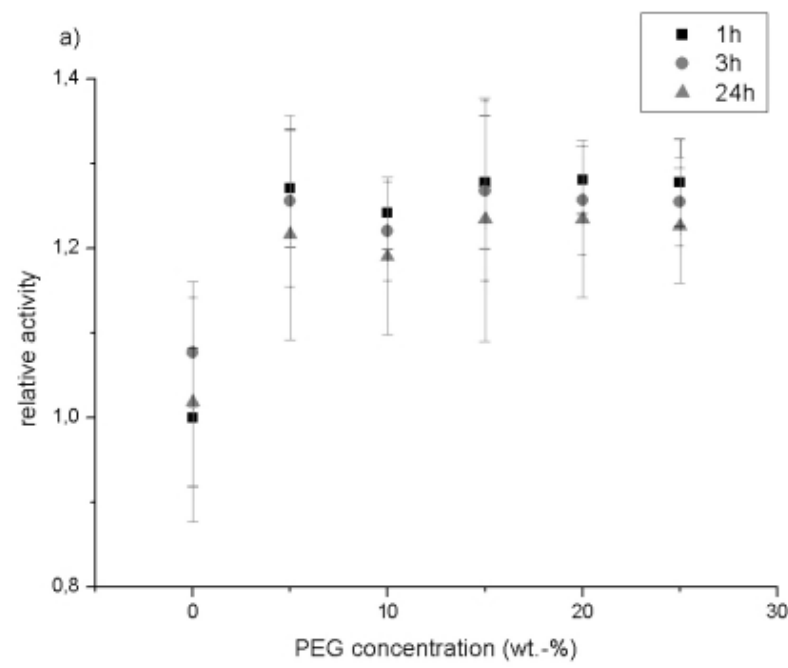

Fig. 2. Influence of PEG on laccase activity

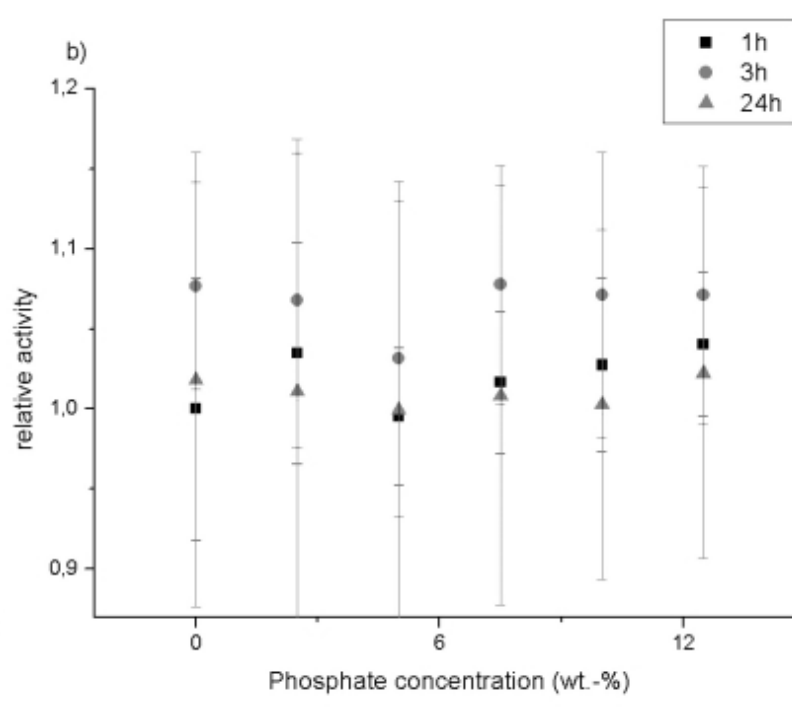

Fig. 3. Influence of phosphate on laccase activity

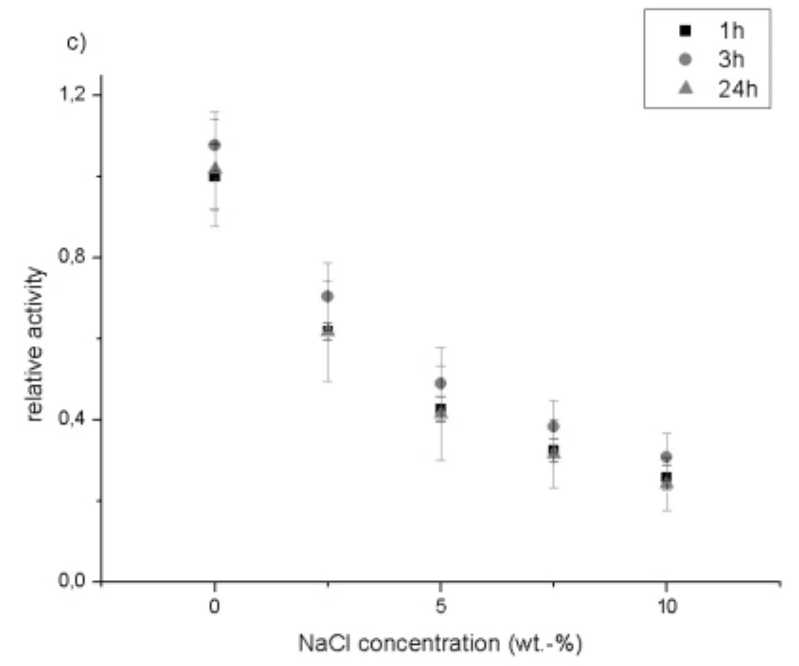

Fig. 4. Influence of $\mathrm{NaCl}$ on laccase activity

The concentrations of PEG3000 in the samples ranged between 0 and 25 wt.-\%. It was found out that the presence of polyethylene glycol in the mixture resulted in a significant increase of enzyme's activity even in small concentrations. As was shown in Fig. 2, the sample containing 5 wt.- $\%$ of the polymer achieved activity almost thirty percent higher than a pure sample. Further addition of PEG3000 did not increase or decrease the enzyme's activity.

To examine phosphate influence on the enzyme's activity, similar mixtures, containing different concentrations of phosphate buffer, were prepared. As seen in Fig. 3, no significant change in activity was observed within the range of 0 to $12.5 \mathrm{wt} .-\%$, therefore it was assumed that the phosphate buffer has no effect over the enzyme's activity.

Since, according to Prinz et al. (2012), halogen salts can sometimes invert the affinity of the enzyme from one phase to another, the influence of various sodium chloride concentrations on the enzyme's activity was also investigated. The concentration range from 0 to $10 \mathrm{wt} .-\%$ of $\mathrm{NaCl}$ was examined. As shown in Fig. 4, the salt turned out to be very disruptive in relation to Cerrena unicolor laccase. Even the addition of $2.5 \mathrm{wt} .-\%$ resulted in a decrease of the enzyme's activity by $40 \%$. At the concentration of 10 wt.- $\%$, the activity dropped below $30 \%$ of the original value. It may have been caused by high 
ionic strength of the solution or the fact that some enzymes are susceptible to deactivation by halide ions.

Furthermore, in all cases, a common trend was observed. After three hours since the mixture preparations, the samples showed higher activity than after one hour, and after a day, the activities dropped below the values of measurements after one hour. The decrease in the enzyme's activity after 24 hours is a result of a well-known phenomenon of enzyme's activity loss over time, caused probably by proteolytic enzymes, since the samples were kept at room temperature.

\subsection{Tie line length}

The first series of experiments was conducted to determine the relationship between tie line lengths and extractive capabilities of the system. Liu et al. (2012) suggest that the increase of TLL reduces water availability and thus increases the hydrophobicity of the system. Since tie line length is not a physical value, but rather a mathematical representation of a relationship between mixing points and their respective phase concentrations, it should be viewed as a general guideline rather than a definite phenomenon governing extraction in aqueous two-phase systems, because depending on the phase forming components and additives introduced to the system, the same tie line lengths can relate to different tie lines. Moreover, the results presented in this paper show a correlation between tie line length and yields or activity partitioning coefficients, but the influence of phase ratio change resulting from the change of tie line lengths would have to be excluded in order to draw causative conclusions.

Table 1. Mixing points chosen for the investigation of line length influence on laccase partitioning

\begin{tabular}{|c|c|c|}
\hline Exp. No & PEG [wt.-\%] & Phosphate [wt.-\%] \\
\hline 1 & 13 & 6 \\
\hline 2 & 14 & 6.46 \\
\hline 3 & 15 & 6.92 \\
\hline 4 & 16 & 7.38 \\
\hline 5 & 17 & 7.85 \\
\hline
\end{tabular}

To determine the effect of tie line lengths on laccase partitioning between phases, a specially designed set of mixtures was prepared. In the chosen set, all mixtures contained the amounts of phase forming components at the same ratio (PEG3000 to Phosphate ratio 13:6), therefore all mixing points lay on one straight line. The mixing points were listed in Table 1.


Fig. 5. Activity partitioning between phases; a) enzyme activities in bottom and top phases as a function of tie line length, b) activity partitioning coefficient as a function of tie line length 
In Fig. 5a, the activities of the enzyme in both phases depending on different tie line lengths are depicted. It can be observed that the increase of tie line length results in lower activities in the bottom phase and higher concentrations in the top phase. In Fig. 5b, a dependence between TLL and the activity partitioning coefficients is presented. A linear decrease can be observed, which can lead to a conclusion that the increase of tie line lengths results in worse partitioning between phases. However, as can be seen in Fig. 6, with the increase of TLL, different mixing points were achieved, which resulted in an increase of phase ratio of this system. Therefore, a causal relationship between tie line length and activity partitioning coefficient cannot be established.

On the other hand, as shown in Fig 7, yields in bottom phase decrease as tie line length increases. Since yield regards enzyme activity in relation to the volume of the phase, it can be concluded that shorter tie lines result in more effective partitioning towards the bottom phase.

From this series of experiments a conclusion can be drawn that Cerrena unicolor laccase shows much higher affinity towards bottom, salt-rich phase, than towards top, polymer-rich phase. The highest activity partitioning coefficient achieved in this batch of experiments was $K_{\text {act }}=11.8$ and the lowest $K_{\text {act }}=5.9$.

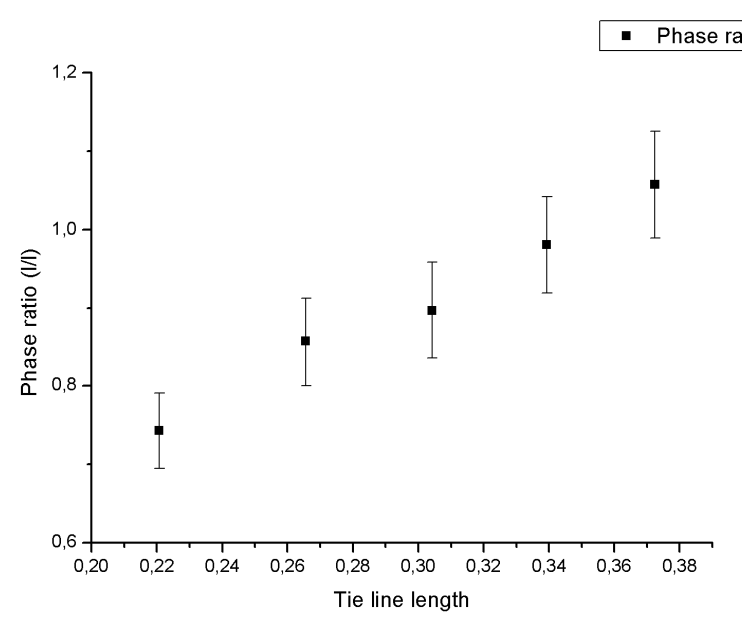

Fig. 6. Phase ratio as a function of tie line length

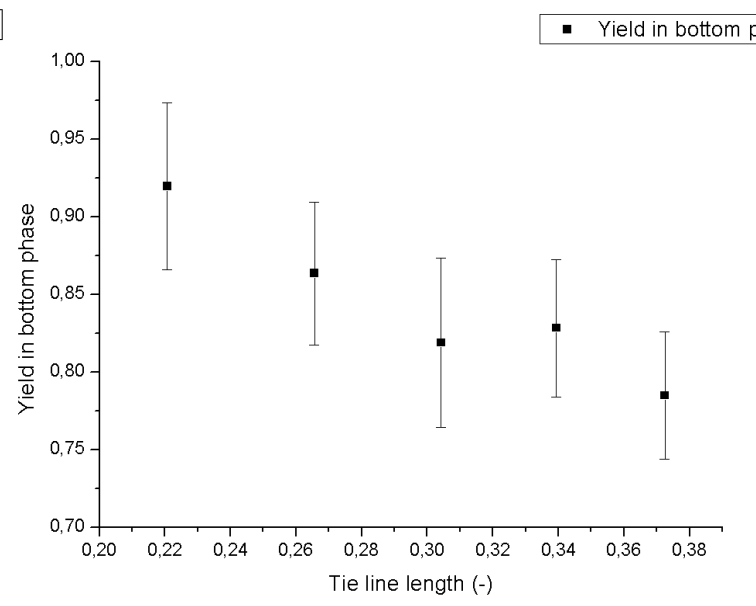

Fig. 7. Tie line length influence on the yields in bottom phase

\subsection{Phase volume ratio}

Another series of experiments regarded the influence of phase ratio on the effectiveness of extraction in aqueous two-phase systems. To investigate this parameter, a single tie line was chosen, and all the considered mixing points lay at the specified tie line. Since the lowest mixing point investigated in section 2 was very close to the critical point, mixtures could turn into one phase. Therefore, the starting mixing point was chosen to consist of 6.5 wt.- $\%$ of Phosphate and 13.84 wt.- $\%$ of PEG3000, to eliminate the threat of falling below the binodal curve. The mixing points were listed in Table 2.

Table 2. Mixing points chosen for phase ratio influence investigation

\begin{tabular}{|c|c|c|}
\hline Exp. No & PEG [wt.-\%] & Phosphate [wt.-\%] \\
\hline 1 & 25.49 & 3 \\
\hline 2 & 19.66 & 4.75 \\
\hline 3 & 13.84 & 6.5 \\
\hline 4 & 8.01 & 8.25 \\
\hline 5 & 2.18 & 10 \\
\hline
\end{tabular}


a)

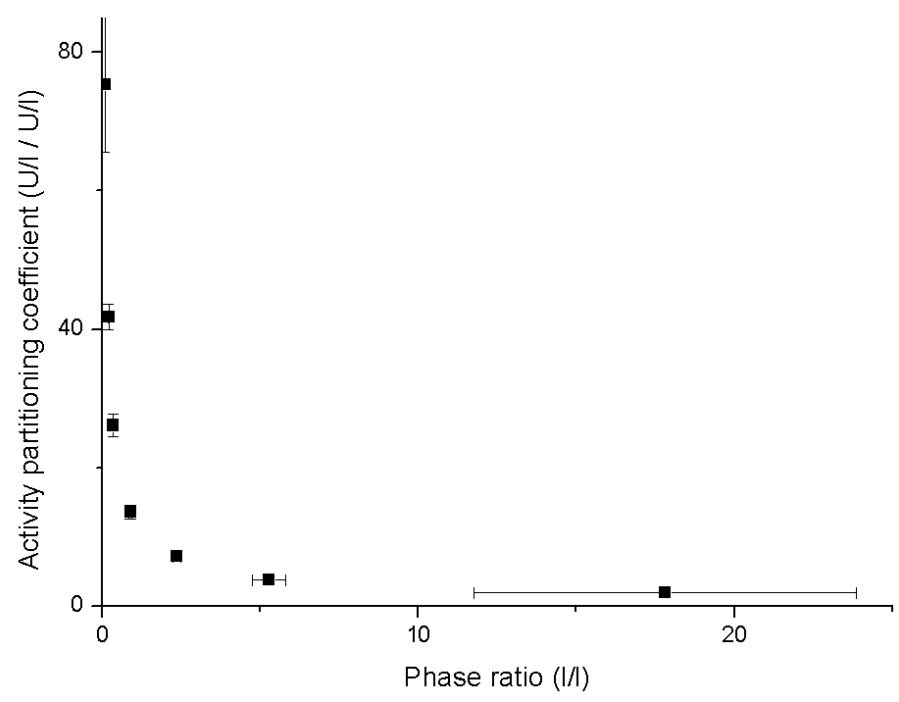

Fig. 8. Phase ratio influence on the activity partitioning coefficient

Fig 8 shows that as the mixing point approaches the binodal curve, the activity partitioning coefficient changes greatly. Proximity to the polymer-rich end of the tie line resulted in very large top phases and small bottom phases, in which the enzyme was concentrated, what therefore resulted in high activity partitioning coefficients. Proximity to salt-rich end of the tie line resulted in highly diluted bottom phases and very low activity partitioning coefficients. The highest activity partitioning coefficient achieved using this method was $K_{\text {act }}=75.4$, and the lowest was equal to $K_{\text {act }}=2.0$.

For very high phase ratios the top phase volumes were very small. Similarly, for very low phase ratios the bottom phase volumes were very small. This resulted in high errors for extreme phase ratios, because small volumetric errors had strong influence on yield and phase ratio values.

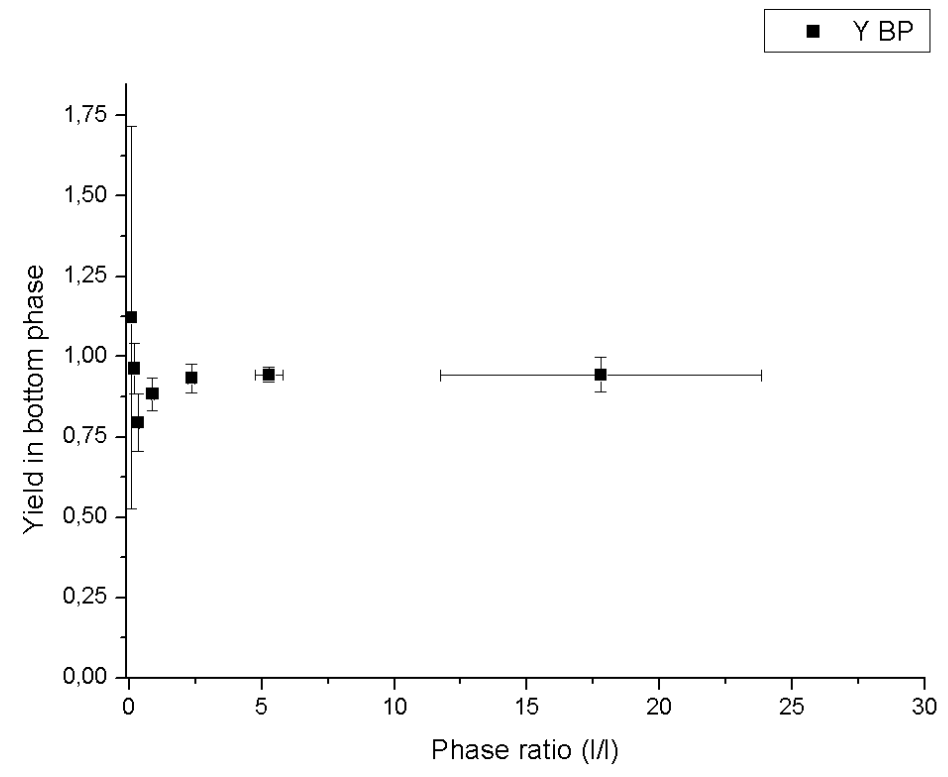

Fig. 9. Phase ratio influence on the yields in bottom phase

In Fig. 9 yields in the bottom phase were shown. According to the obtained results, it can be assumed that larger volumes of phases result in higher amounts of the enzyme diluted in them. Higher phase ratio relates to higher yield in the bottom phase. Although the equilibria ruling this phenomenon are yet to be discovered for aqueous two-phase extraction, Johansson and Reczey (1998) assign it to low 
solubility of proteins in polymer solutions. From the data presented it can be seen that the curve for the bottom phase yield gets very close to its asymptote at phase ratio values around 3 .

\section{4. $\mathrm{NaCl}$ addition}

According to several studies (Gu and Gatz, 2007; Mehrnoush et al., 2012; Prinz et al., 2012), addition of sodium chloride to the extraction mixture may significantly affect the partitioning of the enzyme between phases due to generating an electric potential between the phases.

A set of experiments was performed to investigate its influence on the presented system. Since $\mathrm{NaCl}$ was proven highly disruptive for Cerrena unicolor laccase's activity, only small amounts of the salt ( 0.33 wt.- $\%, 0.66$ wt.- $\%, 1$ wt.- $\%)$ were added to the mixture, to avoid significant deactivation of the enzyme. The rest of the phase forming components and the amount of the supernatant were constant.

In Fig. 10, activities in top and bottom phase were presented. A decrease in the activity in the bottom phase can be observed, and no decrease in the top phase.


Fig. 10. Influence of sodium chloride on laccase's activity;

a) activities in bottom and top phase, b) activity partitioning coefficients

- Phase ratio

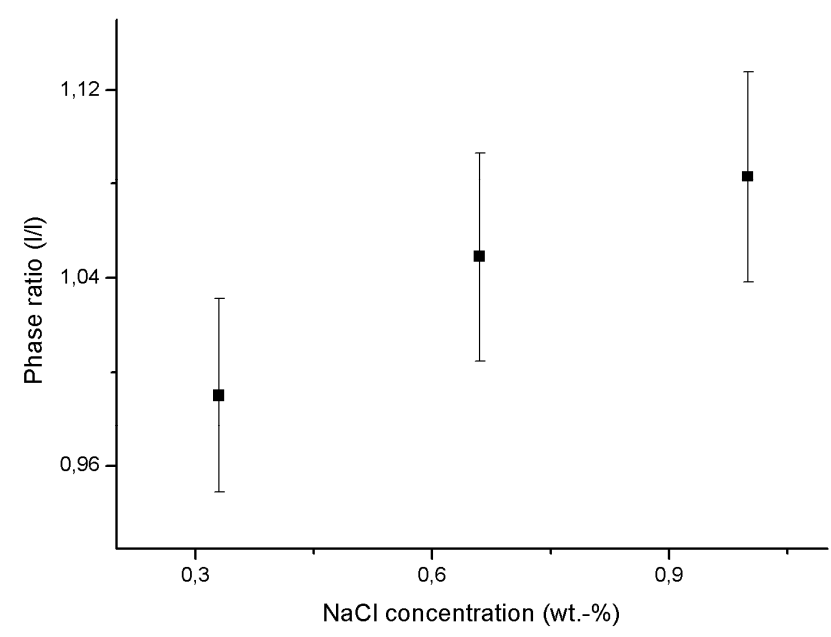

Fig. 11. Sodium chloride addition's influence on the phase ratio 
The addition of $\mathrm{NaCl}$ also influenced the formation of phases, which was presented in Fig. 11. With addition of larger amounts of sodium chloride, higher phase ratio was achieved, which was caused by the larger amount of total salt in the system, which obviously led to larger bottom phases.

No inversion of phase affinity of Cerrena unicolor laccase was observed in the investigated system.

\subsection{Supernatant dilutions}

An additional set of experiments examined whether different amounts of the culture supernatant - and thus different enzyme concentrations - in the system influenced the enzyme's behavior in aqueous two phase extraction. All mixtures contained 6.5 wt.- $\%$ of phosphate and 13.84 wt.- $\%$ of PEG3000. The samples contained supernatant in amounts of $0.8 \mathrm{~g}, 1.6 \mathrm{~g}, 2.4 \mathrm{~g}$ and $3.2 \mathrm{~g}$, and the mixtures were completed to $15 \mathrm{~g}$ with water. In Fig. 12, activities in both phases were presented. In both bottom phase and top phase the activities increased linearly with the increase of the amount of the supernatant, which indicates that supernatant concentration has no influence on activity partitioning equilibria.

Influence of supernatant dilution on bottom phase yields was shown in Fig. 13. The values differed insignificantly within the error range.

According to the data gathered from this set of experiments, it can be concluded that the concentration of the supernatant does not affect the partitioning equilibria between phases.

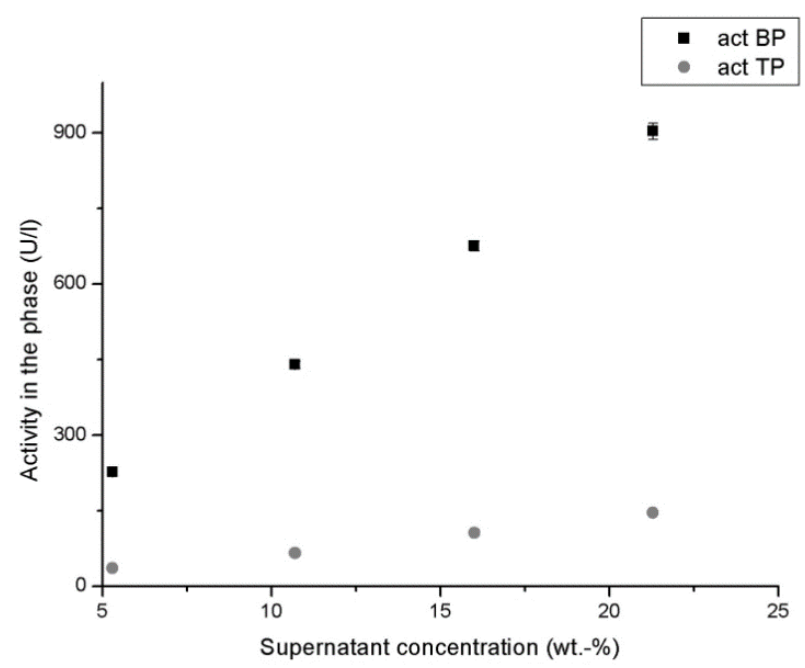

Fig. 12. Supernatant concentrations' influence on the partitioning of laccase activity

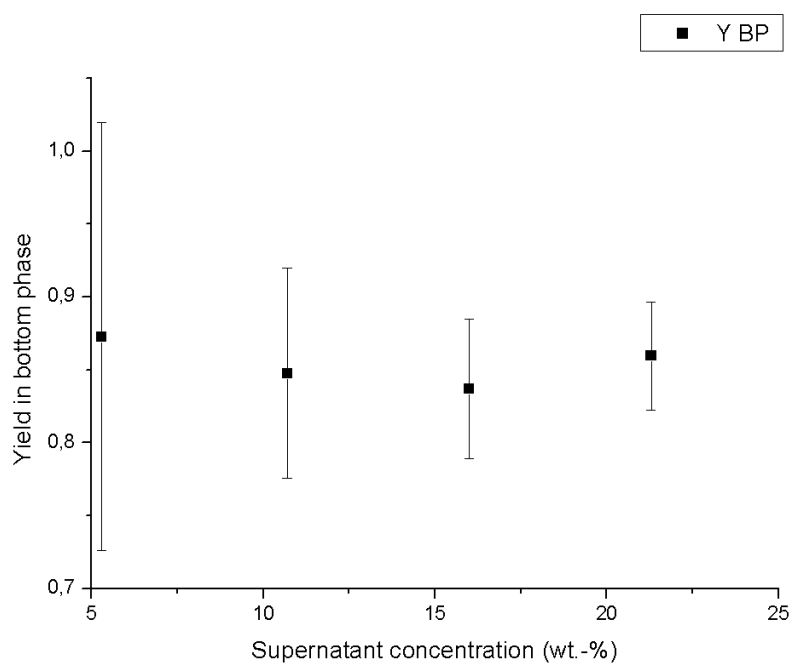

Fig. 13. Supernatant dilutions' influence on the yields in bottom phase

\section{CONCLUSIONS}

The addition of polyethylene glycol 3000 increases the activity of the enzyme by $20 \%$ of the initial value. There was no activity change observed after supplementing the supernatant with phosphate salt. Sodium chloride causes a significant decrease in the laccase's activity. Cerrena unicolor laccase shows affinity towards the bottom, salt-rich, phase of the system. Shorter tie lines lead to more effective partitioning between phases. Mixing points with low concentrations of phosphate salts lead to small bottom phases with large concentrations of the enzyme. No inversion of phase affinity was observed after the addition of sodium chloride into the mixture, although it influenced the forming of the phases. Diluting the supernatant had no influence on laccase's partitioning between phases. 
The research was conducted as a part of a project number UMO-2013/11/B/ST8/00337 financed by the National Science Centre.

\section{SYMBOLS}

act enzymatic activity, $\mathrm{U} / 1$

$d \quad$ thickness of the sample, $\mathrm{mm}$

$D \quad$ dilution coefficient, -

E absorbance, -

$K_{a c t} \quad$ activity partitioning coefficient, -

$t \quad$ time, $\min$

$V \quad$ volume of the phase, $\mathrm{ml}$

$V_{s} \quad$ volume of the supernatant sample, $\mu 1$

$V_{t} \quad$ total volume of the sample, $\mu \mathrm{l}$

$x \quad$ mass fraction, wt- $\%$

Y yield, -

Greek symbols

$\varepsilon \quad$ extinction coefficient, $1 /(\mathrm{mmol} \mathrm{cm})$

$\Phi \quad$ phase ratio, -

\section{Subscripts}

$B P \quad$ bottom phase

TP top phase

\section{REFERENCES}

Azevedo A.M., Rosa P.A.J., Ferreira I.F., Aires-Barros M.R., 2009. Chromatography-free recovery of biopharmaceuticals through aqueous two-phase processing. Trends Biotech., 27, 240-247. DOI: 10.1016/j.tibtech.2009.01.004.

Benavides J., Rito-Palomares M., 2008. Practical experiences from the development of aqueous two-phase processes for the recovery of high value biological products. J. Chem. Technol. Biotechnol., 83, 133-142. DOI: $10.1002 /$ jctb. 1844 .

Bryjak J., Rekuć A., 2009. Effective purification of Cerrena unicolor laccase using microfiltration, ultrafiltration and acetone precipitation. App. Biochem. Biotechnol., 160, 2219-2235. DOI: 10.1007/s12010-009-8791-9.

Claus H., Filip Z., 1997. The evidence for a laccase-like enzyme activity in a Bacillus sphaericus strain. Microbiol. Res., 152, 209-216. DOI: 10.1016/S0944-5013(97)80014-6.

Diamantidis G., Effosse A., Potier P., Bally R., 2001. Purification and characterization of the first bacterial laccase in the rhizospheric bacterium Azospirillum lipoferum. Soil Biol. Biochem., 32, 919-927. DOI: 10.1016/S0038-0717(99)00221-7.

Diamond A., Hsu J., 1989. Fundamental studies of biomolecule partitioning in aqueous two-phase systems. Biotech. Bioeng., 7, 1000-1014. DOI: 10.1002/bit.260340714.

Enebak S.A., Blanchette R.A., 1989. Canker formation and decay in sugar maple and paper birch infected by Cerrena unicolor. Can. J. For. Res., 19, 225-231.

Gu Z., Glatz C.E., 2007. Aqueous two-phase extraction for protein recovery from corn extracts. J. Chromatogr. B, 845, 38-50. DOI: 10.1016/j.jchromb.2006.07.025.

Harvey B.M., Walker J.R.K., 1999. Studies with plant laccases: I. Comparison of plant and fungal laccases. Biochem. Mol. Biol. Biophys., 3, 45-51.

Johansson G., Reczey K., 1998. Concentration and purification of b-glucosidase from Aspergillus niger by using aqueous two-phase partitioning. J. Chromatogr. B, 711, 161-172. DOI: 10.1016/S0378-4347(97)00601-4. 
Kim C.W., Rha C., 2000. Interfacial tension of polyethylene glycol/potassium phosphate aqueous two-phase systems. Phys. Chem. Liq., 38, 25-34. DOI: 10.1080/00319100008045294.

Kula M.R., Kroner K.H., Hustedt H., 1982. Purification of enzymes by liquid-liquid extraction. Adv. Biochem. Eng., 50, 74-118. DOI: 10.1007/3-540-11699-0_11.

Lindeberg G., Holm G., 1952. Occurrence of tyrosinase and laccase in fruit bodies of mycelia of some Hymenomycetes. Physiol. Plant, 5, 100-114. DOI: 10.1111/j.1399-3054.1952.tb08234.x.

Liu Y., Wu Z., Zhang Y., Yuan H., 2012. Partitioning of biomolecules in aqueous two-phase systems of polyethylene glycol and nonionic surfactant. Biochem. Eng. J., 69, 93-99. DOI: 10.1016/j.bej.2012.08.018.

Luterek J., Gianfreda L., Wojtaś-Wasilewska L., Cho M., Rogalski N.S., Jaszek J., Malarczyk M., Staszczak M., Fink-Boots M., Leonowicz A., 1998. Activity of free and immobilized extracellular Cerrena unicolor laccase in water miscible organic solvents. Holzforsch., 52 (6), 589-595.

Majcherczyk A., Johannes Ch., Hüttermann A., 1998. Oxidation of polycyclic aromatic hydrocarbons (PAH) by laccase of trametes versicolor. Enzym. and Microb. Technol., 22, 335-341. DOI: 10.1016/S01410229(97)00199-3.

Mayolo-Deloisa K., Trejo-Hernández M.R., Rito-Palomares M., 2009. Recovery of laccase from the residual compost of Agaricus bisporus in aqueous two-phase systems. Process Biochem., 44, 435-439. DOI: 10.1016/j.procbio.2008.12.010.

Mehrnoush A., Mustafa S., Sarker M.I., Yazid A.M.M., 2012. Optimization of serine protease purification from mango (Mangifera Indica cv. Chokanan) peel in polyethylene glycol/dextran aqueous two phase system. Int. J. Mol. Sci., 13, 3636-3649. DOI: 10.3390/ijms13033636.

Pazuki G.R., Taghikhani V., Vossoughi M., 2009. modeling process partitioning of biomolecules in polymerpolymer and polymer-salt aqueous two-phase systems (ATPS) using and extended excess Gibbs energy model. Z. Phys. Chem., 223, 263-278. DOI: 10.1524/zpch.2009.5421.

Prinz A., Koch K., Górak A., Zeiner T., 2014. Multi-stage laccase extraction and separation using aqueous twophase systems: Experiment and model. Process Biochem., 49, 1020-1031. DOI: 10.1016/j.procbio.2014.03.011.

Prinz A., Zeiner T., Vössing T., Schüttmann I., Zorn H., Górak A., 2012. Experimental investigation of laccase purification using aqueous two-phase extraction. Chem. Eng. Trans., 27, 349-354.

Rajeeva S., Lele. S.S., 2010. Bioprocessing of laccase produced by submerged culture of Ganoderma sp. WR-1. Sep. Purif. Technol., 76, 110-119. DOI: 10.1016/j.seppur.2010.09.027.

Ratanapongleka K., Phetsom J., 2011. extraction in two-phase systems and some properties of laccase from Lentinus polychrous. Eng. Technol., 57, 812-815.

Reschke T., Brandenbusch C., Sadowski G., 2014. Modeling aqueous two-phase systems: I. Polyethylene glycol and inorganic salts as ATPS former. Fluid Phase Equilib., 368, 91-103. DOI: 10.1016/j.fluid.2014.02.016.

Rogalski J., Dawidowicz A., Jóźwik. E., Leonowicz A., 1999. Immobilization of laccase from Cerrena unicolor on controlled porosity glass. J. Mol. Catal. B: Enzym., 6, 29-39. DOI: 10.1016/S1381-1177(98)00117-9.

Salabat A., Moghadam S.T., Far M.R., 2010. Liquid-liquid equilibria of aqueous two-phase systems composed of TritonX-100 and sodium citrate or magnesium sulfate salts. Calphad, 34, 81-83. DOI: 10.1016/j.calphad.2009.12.004.

Silvério S.C., Rodríguez O., Tavares A.P.M., Teixeira J.A., Macedo E.A., 2012. Laccase recovery with aqueous two-phase systems: Enzyme partitioning and stability. J. Mol. Catal. B: Enzym., 87, 37-43. DOI: 10.1016/j.molcatb.2012.10.010.

Yang Y., Ding Y., Liao X., Cai Y., 2013. Purification and characterization of a new laccase from Shiraia sp.SUPER-H168. Process Biochem., 48, 351-357. DOI: 10.1016/j.procbio.2012.12.011.

Xu F., 1996. Oxidation of phenols, anilines, and benzenethiols by fungal laccases: correlation between activity and redox potentials as well as halide inhibition. Biochem., 35, 7608-7614. DOI: 10.1021/bi952971a.

Yoshida H., 1883. Chemistry of lacquer (Urushi). J. Chem. Soc., 43, 472-48.

Received 16 May 2015

Received in revised form 16 March 2016

Accepted 08 April 2016 